\title{
Commutators of Multilinear Singular Integrals on Weighted Herz Spaces
}

\author{
Baozhen Wang and Huimin Wang \\ Department of Mathematics, Huanghuai University, Zhumadian, Henan 463000, China \\ Correspondence should be addressed to Huimin Wang; wanghm1981@yeah.net \\ Received 16 April 2015; Accepted 14 June 2015 \\ Academic Editor: Rodolfo H. Torres
}

Copyright ( $) 2015$ B. Wang and H. Wang. This is an open access article distributed under the Creative Commons Attribution License, which permits unrestricted use, distribution, and reproduction in any medium, provided the original work is properly cited.

Suppose $\vec{b}=\left(b_{1}, \ldots, b_{m}\right) \in(\mathrm{BMO})^{m}, T_{\Pi b}$ is the iterated commutator of $\vec{b}$ and the $m$-linear Calderón-Zygmund operator $T$. The purpose of this paper is to discuss the boundedness properties of $T_{\Pi b}$ on weighted Herz spaces with general Muckenhoupt weights.

\section{Introduction and Results}

In recent years, the study of multilinear integrals has received more attention, which is motivated by not only the generalization of the linear theory but also the natural appearance of singular integral theory. The initial work on the class of multilinear Calderón-Zygmund operators was done by Coifman and Meyer in [1] and was later systematically studied by Grafakos and Torres in [2-4]. More results on the multilinear Calderón-Zygmund operators and their commutators can be found in [5-10].

Let $\mathbb{R}^{n}$ be the $n$-dimensional Euclidean space, and let $\left(\mathbb{R}^{n}\right)^{m}=\mathbb{R}^{n} \times \cdots \times \mathbb{R}^{n}$ be the $m$-fold product space $(m \in$ $\mathbb{N})$. We denote by $\mathcal{S}\left(\mathbb{R}^{n}\right)$ the space of all Schwartz functions on $\mathbb{R}^{n}$ and by $\mathcal{S}^{\prime}\left(\mathbb{R}^{n}\right)$ its dual space, the set of all tempered distributions on $\mathbb{R}^{n}$.

We say that a locally integrable function $K\left(y_{0}, y_{1}, \ldots, y_{m}\right)$ defined away from the diagonal $y_{0}=y_{1}=\cdots=y_{m}$ in $\left(\mathbb{R}^{n}\right)^{m+1}$ is a kernel in the class $m-\operatorname{CZK}(A, \epsilon)$ if it satisfies the size estimate

$$
\left|K\left(y_{0}, y_{1}, \ldots, y_{m}\right)\right| \leq \frac{A}{\left(\sum_{k, l=0}^{m}\left|y_{l}-y_{k}\right|\right)^{m n}}
$$

for some $A>0$ and all $\left(y_{0}, y_{1}, \ldots, y_{m}\right) \in\left(\mathbb{R}^{n}\right)^{m+1}$ with $y_{l} \neq y_{k}$ for some $l, k$. Moreover, assume that for some $\epsilon>0$ and all $0 \leq j \leq m$ it satisfies the smoothness estimates

$$
\begin{aligned}
& \left|K\left(y_{0}, \ldots, y_{j}, \ldots, y_{m}\right)-K\left(y_{0}, \ldots, y_{j}^{\prime}, \ldots, y_{m}\right)\right| \\
& \quad \leq \frac{A\left|y_{j}-y_{j}^{\prime}\right|^{\epsilon}}{\left(\sum_{k, l=0}^{m}\left|y_{l}-y_{k}\right|\right)^{m n+\epsilon}}
\end{aligned}
$$

whenever

$$
\left|y_{j}-y_{j}^{\prime}\right| \leq \frac{1}{2} \max _{0 \leq k \leq m}\left|y_{j}-y_{k}\right|
$$

Let $T$ be a multilinear operator initially defined on the $m$ fold product of Schwartz spaces and taking values into the space of tempered distributions,

$$
T: \mathcal{S}\left(\mathbb{R}^{n}\right) \times \cdots \times \mathcal{S}\left(\mathbb{R}^{n}\right) \longrightarrow \mathcal{S}^{\prime}\left(\mathbb{R}^{n}\right)
$$

We say that $T$ is a $m$-linear Calderón-Zygmund operator if, for some $q_{1}, \ldots, q_{m} \in[1, \infty)$ and $q \in(0, \infty)$ with $1 / q=$ $\sum_{i=1}^{m} 1 / q_{i}$, it extends to a bounded multilinear operator from $L^{q_{1}}\left(\mathbb{R}^{n}\right) \times \cdots \times L^{q_{m}}\left(\mathbb{R}^{n}\right)$ into $L^{q}\left(\mathbb{R}^{n}\right)$ and if there exists a kernel function $K\left(x, y_{1}, \ldots, y_{m}\right)$ in the class $m-\operatorname{CZK}(\mathrm{A}, \epsilon)$, defined 
away from the diagonal $x=y_{1}=\cdots=y_{m}$ in $\left(\mathbb{R}^{n}\right)^{m+1}$, satisfying

$$
\begin{aligned}
& T(\vec{f})(x)=T\left(f_{1}, \ldots, f_{m}\right)(x) \\
& \quad=\int_{\left(\mathbb{R}^{n}\right)^{m}} K\left(x, y_{1}, \ldots, y_{m}\right) \\
& \quad \cdot f_{1}\left(y_{1}\right) \cdots f_{m}\left(y_{m}\right) d y_{1} \cdots d y_{m}
\end{aligned}
$$

for all $x \notin \bigcap_{j=1}^{m} \operatorname{suup} f_{j}$ and $f_{1}, \ldots, f_{m}$ are $C^{\infty}$ functions with compact support.

Given a locally integrable vector function $\vec{b}=\left(b_{1}, \ldots\right.$, $\left.b_{m}\right) \in(\mathrm{BMO})^{m}$, the iterated commutator of $\vec{b}$ and the $m$ linear Calderón-Zygmund operator $T$, denoted by $T_{\Pi b}$, was introduced in [6] and is defined by

$$
T_{\Pi b}(\vec{f})=\left[b_{1}, \ldots,\left[b_{m-1},\left[b_{m}, T\right]_{m}\right]_{m-1} \ldots\right]_{1}(\vec{f}) .
$$

To clarify the notation, if $T$ is associated in the usual way with a Calderón-Zygmund kernel $K$, then at a formal level

$$
\begin{aligned}
& T_{\Pi b}(\vec{f})(x)=\int_{\left(\mathbb{R}^{n}\right)^{m}} \prod_{i=1}^{m}\left(b_{i}(x)-b_{i}\left(y_{i}\right)\right) \\
& \quad \cdot K\left(x, y_{1}, \ldots, y_{m}\right) f_{1}\left(y_{1}\right) \cdots f_{m}\left(y_{m}\right) d y_{1} \cdots d y_{m} .
\end{aligned}
$$

Suppose $T$ is an $m$-linear Calderón-Zygmund operator. Let $\vec{\omega} \in A_{\vec{q}}$, let $\nu_{\vec{\omega}}=\prod_{i=1}^{m} \omega_{i}^{q / q_{i}}$ with $1 / q=1 / q_{1}+\cdots+1 / q_{m}$, and let $\vec{b} \in(\mathrm{BMO})^{m}$. In 2014, Pérez et al. [6] proved that the commutator $T_{\Pi b}$ is bounded from $L^{q_{1}}\left(\omega_{1}\right) \times \cdots \times L^{q_{m}}\left(\omega_{m}\right)$ to $L^{q}\left(\nu_{\vec{\omega}}\right)$.

Let $B_{k}=\left\{x \in \mathbb{R}^{n}:|x| \leq 2^{k}\right\}$ and $C_{k}=B_{k} \backslash B_{k-1}$ for any $k \in \mathbb{Z}$. Denote $\chi_{k}=\chi_{C_{k}}$ for $k \in \mathbb{Z}$, where $\chi_{C_{k}}$ is the characteristic function of the set $C_{k}$. The following weighted Herz space is introduced by Lu and Yang in [11].

Let $\alpha \in \mathbb{R}, 0<p, q<\infty$, and $\omega_{1}, \omega_{2}$ be two weight functions on $\mathbb{R}^{n}$. The homogeneous weighted Herz space $\dot{K}_{q}^{\alpha, p}\left(\omega_{1}, \omega_{2}\right)\left(\mathbb{R}^{n}\right)$ is defined by

$$
\begin{aligned}
\dot{K}_{q}^{\alpha, p} & \left(\omega_{1}, \omega_{2}\right)\left(\mathbb{R}^{n}\right) \\
\quad & =\left\{f \in L_{\mathrm{loc}}^{q}\left(\mathbb{R}^{n} \backslash\{0\}, \omega_{2}\right):\|f\|_{\dot{K}_{q}^{\alpha, p}\left(\omega_{1}, \omega_{2}\right)}<\infty\right\},
\end{aligned}
$$

where

$$
\|f\|_{\dot{K}_{q}^{\alpha, p}\left(\omega_{1}, \omega_{2}\right)}=\left(\sum_{k=-\infty}^{\infty} \omega_{1}\left(B_{k}\right)^{\alpha p / n}\left\|f \chi_{k}\right\|_{L_{\omega_{2}}^{q}\left(\mathbb{R}^{n}\right)}^{p}\right)^{1 / p} .
$$

Obviously, if $\alpha=0$, then $\dot{K}_{q}^{0, q}\left(\omega_{1}, \omega_{2}\right)\left(\mathbb{R}^{n}\right)=L_{\omega_{2}}^{q}\left(\mathbb{R}^{n}\right)$ for any $0<q<\infty$. Thus, the weighted Herz spaces are generalizations of the weighted Lebesgue spaces.

In 2000, Lu et al. in [12] proved boundedness results for sublinear operators on weighted Herz spaces with general Muckenhoupt weights. Recently, many authors considered the boundedness of operators and their commutators on weighted Herz spaces. Wang in [13] proved that the intrinsic square functions are bounded on weighted Herz spaces and in [14] he also obtained the boundedness of the intrinsic square functions on weighted Herz type Hardy spaces. In [15], Kuang considered the boundedness of generalized Hausdorff operators on weighted Herz spaces; Hu et al. in [16] established the weighted boundedness for the commutator of fractional integral operators on Herz spaces. The authors also have studied the boundedness of the multilinear integrals on weighted (unweighted) Herz spaces in [17-19]. In this paper, we focus on the boundedness of the iterated commutator of BMO and the $m$-linear Calderón-Zygmund operator on weighted Herz spaces. Our main results in this paper are formulated as follows.

Theorem 1. Suppose $m \geq 2,\left(b_{1}, \ldots, b_{m}\right) \in(B M O)^{m}, T$ is an $m$-linear Calderón-Zygmund operator, $\nu_{\vec{\omega}}=\prod_{i=1}^{m} \omega_{i}^{q / q_{i}}$, and $v \in A_{r}(1 \leq r<\infty)$. For $i=1, \ldots, m$, let $0<p_{i} \leq \infty$, let $1<q_{i}<\infty$, and let $\omega_{i} \in A_{r_{i}}\left(1 \leq r_{i}<q_{i}\right)$. If $p, q, \alpha$ satisfy

$$
\begin{gathered}
\frac{1}{p}=\sum_{i=1}^{m} \frac{1}{p_{i}}, \\
\frac{1}{q}=\sum_{i=1}^{m} \frac{1}{q_{1}}, \\
\alpha=\sum_{i=1}^{m} \alpha_{i},
\end{gathered}
$$

then $T_{\Pi b}$ is bounded from $\dot{K}_{q_{1}}^{\alpha_{1}, p_{1}}\left(\nu, \omega_{1}\right)\left(\mathbb{R}^{n}\right) \times \cdots \times$ $\dot{K}_{q_{m}}^{\alpha_{m}, p_{m}}\left(\nu, \omega_{m}\right)\left(\mathbb{R}^{n}\right)$ into $\dot{K}_{q}^{\alpha, p}\left(\nu, v_{\vec{\omega}}\right)\left(\mathbb{R}^{n}\right)$ provided that

$$
0<\alpha_{i} r<n\left(1-\frac{r_{i}}{q_{i}}\right)
$$

for $i=1, \ldots, m$.

\section{Some Preliminaries}

A weight $\omega$ is a nonnegative, locally integrable function on $\mathbb{R}^{n}$. The symbol $B=B\left(x_{0}, r_{B}\right)$ denotes the ball with center $x_{0}$, radius $r_{B}$ and for any $\lambda>0, \lambda B$ will stand for the ball $B\left(x_{0}, \lambda r_{B}\right)$. For a given weight function $\omega$ and a measurable set $E$, we also denote the Lebesgue measure of $E$ by $|E|$ and set weighted measure $\omega(E)=\int_{E} \omega(x) d x$.

A weight $\omega$ is said to belong to $A_{q}$ for $1<q<\infty$, if there exists a constant $C$ such that, for every ball $B \subset \mathbb{R}^{n}$,

$$
\left(\frac{1}{|B|} \int_{B} \omega(x) d x\right)\left(\frac{1}{|B|} \int_{B} \omega(x)^{1-q^{\prime}} d x\right)^{q-1} \leq C,
$$

where $q^{\prime}$ is the dual of $q$ such that $1 / q+1 / q^{\prime}=1$. The class $A_{1}$ is defined by replacing the above inequality with

$$
\frac{1}{|B|} \int_{B} w(y) d y \leq C \cdot \underset{x \in B}{\operatorname{essinf}} w(x)
$$

\section{for every ball $B \subset \mathbb{R}^{n}$.}


A weight $\omega$ is said to belong to $A_{\infty}$ if there are positive numbers $C$ and $\delta$ so that

$$
\frac{\omega(E)}{\omega(B)} \leq C\left(\frac{|E|}{|B|}\right)^{\delta}
$$

for all balls $B$ and all measurable $E \subset B$. It is well known that

$$
A_{\infty}=\bigcup_{1 \leq q<\infty} A_{q}
$$

By (12), we have

$$
\left(\int_{B} \omega(x) d x\right)^{1 / q}\left(\int_{B} \omega(x)^{1-q^{\prime}} d x\right)^{1 / q^{\prime}} \leq C|B|
$$

for $1<q<\infty$.

The classical $A_{q}$ weight theory was first introduced by Muckenhoupt in the study of weighted $L^{q}$ boundedness of Hardy-Littlewood maximal function in [20].

Lemma 2 (see $[20,21])$. Suppose $\omega \in A_{q}\left(\mathbb{R}^{n}\right)$ and the following statements hold.

(i) For any $1 \leq q<\infty$, there are positive numbers $C$ and $\delta$ such that

$$
\begin{array}{ll}
\frac{\omega\left(B_{k}\right)}{\omega\left(B_{j}\right)} \leq C 2^{n q(k-j)} & \text { for } k>j, \\
\frac{\omega\left(B_{k}\right)}{\omega\left(B_{j}\right)} \leq C 2^{\delta(k-j)} & \text { for } k<j .
\end{array}
$$

(ii) $A_{q_{1}}\left(\mathbb{R}^{n}\right) \subset A_{q_{2}}\left(\mathbb{R}^{n}\right)$ for any $1 \leq q_{1}<q_{2} \leq \infty$.

(iii) For any $1<q<\infty$, one has $\omega^{1-q^{\prime}} \in A_{q^{\prime}}\left(\mathbb{R}^{n}\right)$. if

A locally integrable function $b$ is said to be in $\operatorname{BMO}\left(\mathbb{R}^{n}\right)$

$$
\sup _{B \subset \mathbb{R}^{n}} \frac{1}{|B|} \int_{B}\left|b(x)-b_{B}\right| d x=\|b\|_{*}<\infty
$$

where $b_{B}=|B|^{-1} \int_{B} b(y) d y$.

Lemma 3 (see [22]). Suppose $\omega \in A_{\infty}$ and $b \in B M O$. Then, for any $1 \leq q<\infty$ and $r_{1}, r_{2}>0$, we have

$$
\begin{aligned}
& \left(\frac{1}{\omega\left(B\left(x_{0}, r_{1}\right)\right)} \int_{B\left(x_{0}, r_{1}\right)}\left|b(x)-b_{B\left(x_{0}, r_{2}\right)}\right|^{q} \omega(x) d x\right)^{1 / q} \\
& \leq C\|b\|_{*}\left(1+\left|\ln \frac{r_{1}}{r_{2}}\right|\right) .
\end{aligned}
$$

Now let us recall the definition of multiple weights. For $m$ exponents $q_{1}, \ldots, q_{m}$, we write $\vec{q}=\left(q_{1}, \ldots, q_{m}\right)$. Let $q_{1}, \ldots, q_{m} \in[1, \infty)$, and let $q \in(0, \infty)$ with $1 / q=\sum_{k=1}^{m} 1 / q_{k}$.
Given $\vec{\omega}=\left(\omega_{1}, \ldots, \omega_{m}\right)$, set $\nu_{\vec{\omega}}=\prod_{i=1}^{m} \omega_{i}^{q / q_{i}}$. We say that $\vec{\omega}$ satisfies the $A_{\vec{q}}$ condition if it satisfies

$$
\begin{aligned}
\sup _{B} & \left(\frac{1}{|B|} \int_{B} \nu_{\vec{\omega}}(x) d x\right)^{1 / q} \\
\cdot & \prod_{i=1}^{m}\left(\frac{1}{|B|} \int_{B} \omega_{i}(x)^{1-q_{i}^{\prime}}(x) d x\right)^{1 / q_{i}^{\prime}}<\infty .
\end{aligned}
$$

When $q_{i}=1,(1 /|B|) \int_{B} \omega_{i}(x)^{1-q_{i}^{\prime}}(x) d x$ is understood as $\left.\operatorname{(inf}_{x \in B} \omega_{i}(x)\right)^{-1}$.

Let $q_{1}, \ldots, q_{m} \in[1, \infty)$, and let $1 / q=\sum_{k=1}^{m} 1 / q_{k}$. By Hölder's inequality,

$$
\nu_{\vec{\omega}}(B)^{1 / q} \leq \prod_{i=1}^{m} \omega_{i}(B)^{1 / q_{i}}
$$

Supposing $\omega_{i} \in A_{q_{i}}, i=1, \ldots, m$, then by (22) and the definition of multiple weights we have $\vec{\omega} \in A_{\vec{q}}$.

Lemma 4 (see [6]). Let $q_{1}, \ldots, q_{m} \in[1, \infty)$, and let $1 / q=$ $\sum_{k=1}^{m} 1 / q_{k}$. Then $\vec{\omega}=\left(\omega_{1}, \ldots, \omega_{m}\right) \in A_{\vec{q}}$ if and only if

$$
\begin{gathered}
v_{\vec{\omega}} \in A_{q m}, \\
\omega_{i}^{1-q_{i}^{\prime}} \in A_{m q_{i}^{\prime}} \text { for } i=1, \ldots, m,
\end{gathered}
$$

where $\nu_{\vec{\omega}}=\prod_{i=1}^{m} \omega_{i}^{q / q_{i}}$ and the condition $\omega_{i}^{1-q_{i}^{\prime}} \in A_{m q_{i}^{\prime}}$ in the case $q_{i}=1$ is understood as $\omega_{i}^{1 / m} \in A_{1}$.

\section{Proof of Theorem 1}

Without loss of generality, we only prove the theorem for the case $m=2$. The method can be extended for any $m$-linear case without any essential difficulty.

Let $v \in A_{r}$, and let $\omega_{l} \in A_{r_{l}}, l=1,2$. If $k>i, k>j$, then from (17) we have

$$
\begin{aligned}
& \frac{\nu\left(B_{k}\right)}{\nu\left(B_{i}\right)} \leq C 2^{(k-i) n r,} \\
& \frac{\nu\left(B_{k}\right)}{\nu\left(B_{j}\right)} \leq C 2^{(k-j) n r}, \\
& \frac{\omega_{1}\left(B_{k}\right)}{\omega_{1}\left(B_{i}\right)} \leq C 2^{(k-i) n r_{1},} \\
& \frac{\omega_{2}\left(B_{k}\right)}{\omega_{2}\left(B_{j}\right)} \leq C 2^{(k-j) n r_{2}} .
\end{aligned}
$$


If $k<i, k<j$, then from (18) we have

$$
\begin{aligned}
& \frac{v\left(B_{k}\right)}{v\left(B_{i}\right)} \leq C 2^{(k-i) \delta}, \\
& \frac{v\left(B_{k}\right)}{v\left(B_{j}\right)} \leq C 2^{(k-j) \delta}, \\
& \frac{\omega_{1}\left(B_{k}\right)}{\omega_{1}\left(B_{i}\right)} \leq C 2^{(k-i) \delta_{1},} \\
& \frac{\omega_{2}\left(B_{k}\right)}{\omega_{2}\left(B_{j}\right)} \leq C 2^{(k-j) \delta_{2}} .
\end{aligned}
$$

We have the following conclusions.

Theorem 5. Suppose $\left(b_{1}, b_{2}\right) \in(B M O)^{2}, T$ is an 2-linear Calderón-Zygmund operator, and $v \in A_{r}(1 \leq r<\infty)$. For $i=1,2$, let $1<q_{i}<\infty$, and let $\omega_{i} \in A_{r_{i}}\left(1 \leq r_{i}<q_{i}\right)$. If $q$, $\alpha$ satisfy $1 / q=1 / q_{1}+1 / q_{2}$ and $\alpha=\alpha_{1}+\alpha_{2}$, then

$$
\begin{aligned}
& v\left(B_{k}\right)^{\alpha / n}\left\|T_{\Pi b}\left(f \chi_{i}, g \chi_{j}\right) \chi_{k}\right\|_{L^{q}\left(v_{\vec{\omega}}\right)} \\
& \leq C\left\|b_{1}\right\|_{*} D_{1}(k, i) v\left(B_{i}\right)^{\alpha_{1} / n}\left\|f \chi_{i}\right\|_{L^{q_{1}}\left(\omega_{1}\right)} \\
& \quad \times\left\|b_{2}\right\|_{*} D_{2}(k, j) v\left(B_{j}\right)^{\alpha_{2} / n}\left\|g \chi_{j}\right\|_{L^{q_{2}}\left(\omega_{2}\right)},
\end{aligned}
$$

where $\nu_{\vec{\omega}}=\prod_{i=1}^{2} \omega_{i}^{q / q_{i}}$, and

$$
D_{l}(k, i)
$$

$$
= \begin{cases}(k-i) 2^{(k-i)\left(\alpha_{1} r+n r_{1} / q_{1}-n\right)} & \text { if } i \leq k-2 ; \\ 1 & \text { if } k-1 \leq i \leq k-1 \\ (i-k) 2^{(k-i)\left(\delta \alpha_{1} / n+\delta_{1} / q_{1}\right)} & \text { if } i \geq k+2\end{cases}
$$

for $l=1,2$.

Proof. When $k-1 \leq i, j \leq k+1$, then, by the boundedness of $T_{\Pi b}$ from $L^{q_{1}}\left(\omega_{1}\right) \times L^{q_{2}}\left(\omega_{2}\right)$ to $L^{q}\left(\nu_{\vec{\omega}}\right)$ and $\nu\left(B_{k}\right) \sim v\left(B_{i}\right) \sim$ $\nu\left(B_{j}\right)$, we obtain

$$
\begin{gathered}
v\left(B_{k}\right)^{\alpha / n}\left\|T_{\Pi b}\left(f \chi_{i}, g \chi_{j}\right) \chi_{k}\right\|_{L^{q}\left(v_{\vec{\omega}}\right)} \\
\leq C\left\|b_{1}\right\|_{*} v\left(B_{i}\right)^{\alpha_{1} / n}\left\|f \chi_{i}\right\|_{L^{q_{1}\left(\omega_{1}\right)}} \\
\quad \times\left\|b_{2}\right\|_{*} \nu\left(B_{j}\right)^{\alpha_{2} / n}\left\|g \chi_{j}\right\|_{L^{q_{2}}\left(\omega_{2}\right)} .
\end{gathered}
$$

This means $D_{1}(k, i)=D_{2}(k, j)=1$ if $k-1 \leq i, j \leq k+1$.

In the other case, we see that

$$
\left|x-y_{1}\right|+\left|x-y_{2}\right| \sim 2^{\max \{i, j, k\}}
$$

for $x \in C_{k}, y_{1} \in C_{i}, y_{2} \in C_{j}$.

Taking

$$
\begin{aligned}
& \lambda_{1 i}=\left(b_{1}\right)_{B_{i}}=\frac{1}{\left|B_{i}\right|} \int_{B_{i}} b_{1}(x) d x, \\
& \lambda_{2 j}=\left(b_{2}\right)_{B_{j}}=\frac{1}{\left|B_{j}\right|} \int_{B_{j}} b_{2}(x) d x,
\end{aligned}
$$

then

$$
\begin{aligned}
T_{\Pi b}( & \left.f \chi_{i}, g \chi_{j}\right)(x) \\
= & \left(b_{1}(x)-\lambda_{1 i}\right)\left(b_{2}(x)-\lambda_{2 j}\right) T\left(f \chi_{i}, g \chi_{j}\right) \\
& -\left(b_{1}(x)-\lambda_{1 i}\right) T\left(f \chi_{i},\left(b_{2}-\lambda_{2 j}\right) g \chi_{j}\right) \\
& -\left(b_{2}(x)-\lambda_{2 j}\right) T\left(\left(b_{1}-\lambda_{1 i}\right) f \chi_{i}, g \chi_{j}\right)(x) \\
& +T\left(\left(b_{1}-\lambda_{1 i}\right) f \chi_{i},\left(b_{2}-\lambda_{2 j}\right) g \chi_{j}\right)(x) \\
= & L_{1}(x)+L_{2}(x)+L_{3}(x)+L_{4}(x) .
\end{aligned}
$$

Thus

$$
\begin{aligned}
& \nu\left(B_{k}\right)^{\alpha / n}\left\|T_{\Pi b}\left(f \chi_{i}, g \chi_{j}\right) \chi_{k}\right\|_{L^{q}\left(v_{\vec{\omega}}\right)} \\
& \leq \sum_{m=1}^{4} v\left(B_{k}\right)^{\alpha / n}\left\|\left(L_{m}\right) \chi_{k}\right\|_{L^{q}\left(v_{\vec{\omega}}\right)}=\sum_{m=1}^{4} J_{m} .
\end{aligned}
$$

Now, we will estimate each $J_{m}(m=1,2,3,4)$, separately. Applying (29),

$$
\begin{aligned}
& \left|L_{1}(x)\right| \\
& \quad=\left|\left(b_{1}(x)-\lambda_{1 i}\right)\left(b_{2}(x)-\lambda_{2 j}\right) T\left(f \chi_{i}, g \chi_{j}\right)(x)\right| \\
& \quad \leq C 2^{-2 \max \{i, j, k\} n}\left|\left(b_{1}(x)-\lambda_{1 i}\right)\left(b_{2}(x)-\lambda_{2 j}\right)\right| \\
& \quad \cdot\left\|f \chi_{i}\right\|_{L^{1}}\left\|g \chi_{j}\right\|_{L^{1}} .
\end{aligned}
$$

By Hölder's inequality and (16),we have

$$
\begin{aligned}
& \left|L_{1}(x)\right| \leq C 2^{-2 \max \{i, j, k\} n+i n+j n}\left|b_{1}(x)-\lambda_{1 i}\right| \\
& \cdot\left|b_{2}(x)-\lambda_{2 j}\right| \times\left\|f \chi_{i}\right\|_{L^{q_{1}\left(\omega_{1}\right)}}\left\|g \chi_{j}\right\|_{L^{q_{2}\left(\omega_{2}\right)}} \\
& \cdot\left(\int_{B_{i}} \omega_{1}(x)^{1-q_{1}^{\prime}} d x\right)^{1 / q_{1}^{\prime}}\left(\int_{B_{j}} \omega_{2}(x)^{1-q_{2}^{\prime}} d x\right)^{1 / q_{2}^{\prime}} \\
& \leq C 2^{-2 \max \{i, j, k\} n+i n+j n}\left|b_{1}(x)-\lambda_{1 i}\right|\left|b_{2}(x)-\lambda_{2 j}\right| \\
& \quad \times\left\|f \chi_{i}\right\|_{L^{q_{1}\left(\omega_{1}\right)}}\left\|g \chi_{j}\right\|_{L^{q_{2}\left(\omega_{2}\right)}} \omega_{1}\left(B_{i}\right)^{-1 / q_{1}} \omega_{2}\left(B_{j}\right)^{-1 / q_{2}} .
\end{aligned}
$$

Then

$$
\begin{aligned}
& v\left(B_{k}\right)^{\alpha / n}\left\|\left(L_{1}\right) \chi_{k}\right\|_{L^{q}\left(v_{\vec{\omega}}\right)} \\
& \leq C 2^{-2 \max \{i, j, k\} n+i n+j n} v\left(B_{i}\right)^{\alpha_{1} / n}\left\|f \chi_{i}\right\|_{L^{q_{1}}\left(\omega_{1}\right)} \\
& \cdot v\left(B_{j}\right)^{\alpha_{2} / n}\left\|g \chi_{j}\right\|_{L^{q_{2}\left(\omega_{2}\right)}} \times\left(\frac{v\left(B_{k}\right)}{v\left(B_{i}\right)}\right)^{\alpha_{1} / n} \\
& \cdot\left(\frac{v\left(B_{k}\right)}{v\left(B_{j}\right)}\right)^{\alpha_{2} / n} \omega_{1}\left(B_{i}\right)^{-1 / q_{1}} \omega_{2}\left(B_{j}\right)^{-1 / q_{2}}
\end{aligned}
$$


Journal of Function Spaces

5

$$
\begin{aligned}
& \times\left(\int_{B_{k}}\left|\left(b_{1}(x)-\lambda_{1 i}\right)\left(b_{2}(x)-\lambda_{2 j}\right)\right|^{q}\right. \\
& \left.\cdot \nu_{\vec{\omega}}(x) d x\right)^{1 / q} .
\end{aligned}
$$

By Lemma 4 we know $v_{\vec{\omega}} \in A_{\infty}$. If $q \geq 1 / 2$, then by Hölder's inequality, Lemma 3, and (22) we have

$$
\begin{aligned}
& \left(\int_{B_{k}}\left|\left(b_{1}(x)-\lambda_{1 i}\right)\left(b_{2}(x)-\lambda_{2 j}\right)\right|^{q} \nu_{\vec{\omega}}(x) d x\right)^{1 / q} \\
& \quad \leq\left(\int_{B_{k}}\left|b_{1}(x)-\lambda_{1 i}\right|^{2 q} \nu_{\vec{\omega}}(x) d x\right)^{1 / 2 q} \\
& \cdot\left(\int_{B_{k}}\left|b_{2}(x)-\lambda_{1 j}\right|^{2 q} \nu_{\vec{\omega}}(x) d x\right)^{1 / 2 q} \leq C\left\|b_{1}\right\|_{*} \\
& \cdot\left\|b_{2}\right\|_{*}|k-i||k-j| \nu_{\vec{\omega}}\left(B_{k}\right)^{1 / q} \leq C\left\|b_{1}\right\|_{*}\left\|b_{2}\right\|_{*} \\
& \cdot|k-i||k-j| \omega_{1}\left(B_{k}\right)^{1 / q_{1}} \omega_{2}\left(B_{k}\right)^{1 / q_{2}} .
\end{aligned}
$$

If $0<q<1 / 2$, then by Hölder's and Lemma 3 we have

$$
\begin{aligned}
& \left(\int_{B_{k}}\left|\left(b_{1}(x)-\lambda_{1 i}\right)\left(b_{2}(x)-\lambda_{2 j}\right)\right|^{q} \nu_{\vec{\omega}}(x) d x\right)^{1 / q} \\
& \quad \leq\left(\int_{B_{k}}\left|b_{1}(x)-\lambda_{1 i}\right| \nu_{\vec{\omega}}(x) d x\right) \\
& \cdot\left(\int_{B_{k}}\left|b_{2}(x)-\lambda_{1 j}\right|^{q /(1-q)} \nu_{\vec{\omega}}(x) d x\right)^{1 / q-1} \\
& \leq C\left\|b_{1}\right\|_{*}|k-i| \nu_{\vec{\omega}}\left(B_{k}\right) \\
& \cdot\left(\int_{B_{k}}\left|b_{2}(x)-\lambda_{2 j}\right| \nu_{\vec{\omega}}(x) d x\right) \nu_{\vec{\omega}}\left(B_{k}\right)^{1 / q-2} \\
& \leq C\left\|b_{1}\right\|_{*}\left\|b_{2}\right\|_{*}|k-i||k-j| \omega_{1}\left(B_{k}\right)^{1 / q_{1}} \omega_{2}\left(B_{k}\right)^{1 / q_{2}} .
\end{aligned}
$$

Thus

$$
\begin{aligned}
& v\left(B_{k}\right)^{\alpha / n}\left\|\left(L_{1}\right) \chi_{k}\right\|_{L^{q}\left(v_{\vec{\omega}}\right)} \leq C\left\|b_{1}\right\|_{*}|k-i| \\
& \cdot 2^{-\max \{i, k\} n+i n} \nu\left(B_{i}\right)^{\alpha_{1} / n}\left\|f \chi_{i}\right\|_{L^{q_{1}\left(\omega_{1}\right)}}\left(\frac{\nu\left(B_{k}\right)}{v\left(B_{i}\right)}\right)^{\alpha_{1} / n} \\
& \cdot\left(\frac{\omega_{1}\left(B_{k}\right)}{\omega_{1}\left(B_{i}\right)}\right)^{1 / q_{1}} \times\left\|b_{2}\right\|_{*}|k-j| \\
& \cdot 2^{-\max \{j, k\} n+j n} \nu\left(B_{j}\right)^{\alpha_{2} / n}\left\|g \chi_{j}\right\|_{L^{q_{2}\left(\omega_{2}\right)}}\left(\frac{\nu\left(B_{k}\right)}{\nu\left(B_{j}\right)}\right)^{\alpha_{2} / n} \\
& \cdot\left(\frac{\omega_{2}\left(B_{k}\right)}{\omega_{2}\left(B_{j}\right)}\right)^{1 / q_{2}} .
\end{aligned}
$$

When $i \leq k-2, j \leq k-2$. Then by (38) and (24), we get

$$
\begin{aligned}
& v\left(B_{k}\right)^{\alpha / n}\left\|\left(L_{1}\right) \chi_{k}\right\|_{L^{q}\left(v_{\vec{\omega}}\right)} \leq C\left\|b_{1}\right\|_{*}(k-i) \\
& \cdot 2^{(k-i)\left(\alpha_{1} r+n r_{1} / q_{1}-n\right)} v\left(B_{i}\right)^{\alpha_{1} / n}\left\|f \chi_{i}\right\|_{L^{q_{1}}\left(\omega_{1}\right)} \times\left\|b_{2}\right\|_{*} \\
& \cdot(k-j) 2^{(k-j)\left(\alpha_{2} r+n r_{1} / q_{2}-n\right)} v\left(B_{i}\right)^{\alpha_{2} / n}\left\|f \chi_{i}\right\|_{L^{q_{2}}\left(\omega_{2}\right)} .
\end{aligned}
$$

When $i \geq k+2, j \geq k+2$, by (38) and (25), we get

$$
\begin{gathered}
v\left(B_{k}\right)^{\alpha / n}\left\|T_{\Pi b}\left(f \chi_{i}, g \chi_{j}\right) \chi_{k}\right\|_{L^{q}\left(v_{\vec{\omega}}\right)} \leq C\left\|b_{1}\right\|_{*}(i-k) \\
\cdot 2^{(k-i)\left(\alpha_{1} \delta / n+\delta_{1} / q_{1}\right)} v\left(B_{i}\right)^{\alpha_{1} / n}\left\|f \chi_{i}\right\|_{L^{q_{1}}\left(\omega_{1}\right)} \times\left\|b_{2}\right\|_{*} \\
\cdot(j-k) 2^{(k-j)\left(\alpha_{2} \delta / n+\delta_{2} / q_{2}\right)} v\left(B_{j}\right)^{\alpha_{2} / n}\left\|g \chi_{i}\right\|_{L^{q_{2}}\left(\omega_{2}\right)} .
\end{gathered}
$$

When $i \leq k-2, j \geq k+2$. Then by (38) and (24)-(25) we get

$$
\begin{gathered}
v\left(B_{k}\right)^{\alpha / n}\left\|T_{\Pi b}\left(f \chi_{i}, g \chi_{j}\right) \chi_{k}\right\|_{L^{q}\left(v_{\vec{\omega}}\right)} \leq C\left\|b_{1}\right\|_{*}(k-i) \\
\cdot 2^{(k-i)\left(\alpha_{1} r+n r_{1} / q_{1}-n\right)} v\left(B_{i}\right)^{\alpha_{1} / n}\left\|f \chi_{i}\right\|_{L^{q_{1}\left(\omega_{1}\right)}} \times\left\|b_{2}\right\|_{*} \\
\cdot(j-k) 2^{(k-j)\left(\alpha_{2} \delta / n+\delta_{2} / q_{2}\right)} v\left(B_{j}\right)^{\alpha_{2} / n}\left\|g \chi_{j}\right\|_{L^{q_{2}\left(\omega_{2}\right)}} .
\end{gathered}
$$

By symmetry, when $j \leq k-2, i \geq k+2$, we have

$$
\begin{gathered}
\nu\left(B_{k}\right)^{\alpha / n}\left\|T_{\Pi b}\left(f \chi_{i}, g \chi_{j}\right) \chi_{k}\right\|_{L^{q}\left(v_{\vec{\omega}}\right)} \leq C\left\|b_{1}\right\|_{*}(i-k) \\
\cdot 2^{(k-i)\left(\alpha_{1} \delta / n+\delta_{1} / q_{1}\right)} v\left(B_{i}\right)^{\alpha_{1} / n}\left\|f \chi_{i}\right\|_{L^{q_{1}}\left(\omega_{1}\right)} \times\left\|b_{2}\right\|_{*} \\
\cdot(k-j) 2^{(k-j)\left(\alpha_{2} r+n r_{1} / q_{2}-n\right)} v\left(B_{i}\right)^{\alpha_{2} / n}\left\|f \chi_{i}\right\|_{L^{q_{2}}\left(\omega_{2}\right)} .
\end{gathered}
$$

When $i \leq k-2, k-1 \leq j \leq k+1$. Noting that $\nu\left(B_{k}\right) \sim$ $\nu\left(B_{j}\right), \omega_{2}\left(B_{k}\right) \sim \omega_{2}\left(B_{j}\right)$, then

$$
\begin{aligned}
& \nu\left(B_{k}\right)^{\alpha / n}\left\|T_{\Pi b}\left(f \chi_{i}, g \chi_{j}\right) \chi_{k}\right\|_{L^{q}\left(\nu_{\vec{\omega}}\right)} \leq C\left\|b_{1}\right\|_{*}(k-i) \\
& \cdot 2^{(k-i)\left(\alpha_{1} r+n r_{1} / q_{1}-n\right)} v\left(B_{i}\right)^{\alpha_{1} / n}\left\|f \chi_{i}\right\|_{L^{q_{1}}\left(\omega_{1}\right)} \times\left\|b_{2}\right\|_{*} \\
& \cdot v\left(B_{j}\right)^{\alpha_{2} / n}\left\|g \chi_{j}\right\|_{L^{q_{2}\left(\omega_{2}\right)}} .
\end{aligned}
$$

Similar to the estimates (43), we can verify (26) in the following case: $k-1 \leq i \leq k+1, j \leq k-2 ; k-1 \leq i \leq$ $k+1, j>k+1$ and $i>k+1, k-1 \leq j \leq k+1$. Thus we obtain

$$
\begin{aligned}
& v\left(B_{k}\right)^{\alpha / n}\left\|\left(L_{1}\right) \chi_{k}\right\|_{L^{q}\left(v_{\vec{\omega}}\right)} \\
& \leq C\left\|b_{1}\right\|_{*} D_{1}(k, i) v\left(B_{i}\right)^{\alpha_{1} / n}\left\|f \chi_{i}\right\|_{L^{q_{1}}\left(\omega_{1}\right)} \\
& \quad \times\left\|b_{2}\right\|_{*} D_{2}(k, j) v\left(B_{j}\right)^{\alpha_{2} / n}\left\|g \chi_{j}\right\|_{L^{q_{2}}\left(\omega_{2}\right)} .
\end{aligned}
$$


Applying (29) and Hölder's inequality,

$$
\begin{aligned}
& \left|L_{2}(x)\right|=\left|\left(b_{1}(x)-\lambda_{1 i}\right) T\left(f \chi_{i},\left(b_{2}-\lambda_{2 j}\right) g \chi_{j}\right)\right| \\
& \quad \leq C 2^{-2 \max \{i, j, k\} n}\left|b_{1}(x)-\lambda_{1 i}\right|\left\|f \chi_{i}\right\|_{L^{1}} \\
& \cdot\left\|\left(b_{2}-\lambda_{2 j}\right) g \chi_{j}\right\|_{L^{1}} \leq C 2^{-2 \max \{i, j, k\} n}\left|b_{1}(x)-\lambda_{1 i}\right| \\
& \cdot\left\|f \chi_{i}\right\|_{L^{q_{1}}\left(\omega_{1}\right)}\left(\int_{B_{i}} \omega_{1}(x)^{1-q_{1}^{\prime}} d x\right)^{1 / q_{1}^{\prime}} \times\left\|g \chi_{j}\right\|_{L^{q_{2}}\left(\omega_{2}\right)} \\
& \cdot\left(\int_{B_{j}}\left|b_{2}(x)-\lambda_{2 j}\right|^{q_{2}^{\prime}} \omega_{2}(x)^{1-q_{2}^{\prime}} d x\right)^{1 / q_{2}^{\prime}} .
\end{aligned}
$$

By Lemma 2 we know $\omega_{2}^{1-q_{2}^{\prime}} \in A_{q_{2}^{\prime}}$. Then, by Lemma 3, we have

$$
\begin{gathered}
\left(\int_{B_{j}}\left|b_{2}(x)-\lambda_{2 j}\right|^{q_{2}^{\prime}} \omega_{2}(x)^{1-q_{2}^{\prime}} d x\right)^{1 / q_{2}^{\prime}} \\
\leq C\left\|b_{2}\right\|_{*}\left(\int_{B_{j}} \omega_{2}(x)^{1-q_{2}^{\prime}} d x\right)^{1 / q_{2}^{\prime}} .
\end{gathered}
$$

Then, by (46) and (16), we have

$$
\begin{aligned}
& \left|L_{2}(x)\right| \leq C\left\|b_{2}\right\|_{*} 2^{-2 \max \{i, j, k\} n}\left|b_{1}(x)-\lambda_{1 i}\right| \\
& \cdot\left\|f \chi_{i}\right\|_{L^{q_{1}}\left(\omega_{1}\right)}\left(\int_{B_{i}} \omega_{1}(x)^{1-q_{1}^{\prime}} d x\right)^{1 / q_{1}^{\prime}} \times\left\|g \chi_{j}\right\|_{L^{q_{2}}\left(\omega_{2}\right)} \\
& \cdot\left(\int_{B_{j}} \omega_{2}(x)^{1-q_{2}^{\prime}} d x\right)^{1 / q_{2}^{\prime}} \\
& \leq C 2^{-\max \{i, k\} n+i n}\left|b_{1}(x)-\lambda_{1 i}\right|\left\|f \chi_{i}\right\|_{L^{q_{1}}\left(\omega_{1}\right)} \\
& \cdot \omega_{1}\left(B_{i}\right)^{-1 / q_{1}} \times\left\|b_{2}\right\|_{*}|k-j| \\
& \cdot 2^{-\max \{j, k\} n+j n}\left\|g \chi_{j}\right\|_{L^{q_{2}}\left(\omega_{2}\right)} \omega_{2}\left(B_{j}\right)^{-1 / q_{2}} .
\end{aligned}
$$

Note that, for $0<q<\infty$,

$$
\begin{aligned}
& \left(\int_{B_{k}}\left|b_{1}(x)-\lambda_{1 i}\right|^{q} \nu_{\vec{\omega}}(x) d x\right)^{1 / q} \\
& \quad \leq C\left\|b_{1}\right\|_{*}|k-i| \nu_{\vec{\omega}}\left(B_{k}\right)^{1 / q} \\
& \quad \leq C\left\|b_{1}\right\|_{*}|k-i| \omega_{1}\left(B_{k}\right)^{1 / q_{1}} \omega_{2}\left(B_{k}\right)^{1 / q_{2}} .
\end{aligned}
$$

Thus,

$$
\begin{aligned}
& \nu\left(B_{k}\right)^{\alpha / n}\left\|\left(L_{2}\right) \chi_{k}\right\|_{L^{q}\left(\nu_{\vec{\omega}}\right)} \leq C\left\|b_{1}\right\|_{*}|k-i| \\
& \cdot 2^{-\max \{i, k\} n+i n} \nu\left(B_{i}\right)^{\alpha_{1} / n}\left\|f \chi_{i}\right\|_{L^{q_{1}\left(\omega_{1}\right)}}\left(\frac{\nu\left(B_{k}\right)}{\nu\left(B_{i}\right)}\right)^{\alpha_{1} / n}
\end{aligned}
$$

$$
\begin{aligned}
& \cdot\left(\frac{\omega_{1}\left(B_{k}\right)}{\omega_{1}\left(B_{i}\right)}\right)^{1 / q_{1}} \times\left\|b_{2}\right\|_{*}|k-j| \\
& \cdot 2^{-\max \{j, k\} n+j n} \nu\left(B_{j}\right)^{\alpha_{2} / n}\left\|g \chi_{j}\right\|_{L^{q_{2}\left(\omega_{2}\right)}}\left(\frac{\nu\left(B_{k}\right)}{\nu\left(B_{j}\right)}\right)^{\alpha_{2} / n} \\
& \cdot\left(\frac{\omega_{2}\left(B_{k}\right)}{\omega_{2}\left(B_{j}\right)}\right)^{1 / q_{2}} \cdot
\end{aligned}
$$

Similar to the estimates of (44), we get

$$
\begin{aligned}
& \nu\left(B_{k}\right)^{\alpha / n}\left\|\left(L_{2}\right) \chi_{k}\right\|_{L^{q}\left(v_{\vec{\omega}}\right)} \\
& \leq C\left\|b_{1}\right\|_{*} D_{1}(k, i) v\left(B_{i}\right)^{\alpha_{1} / n}\left\|f \chi_{i}\right\|_{L^{q_{1}\left(\omega_{1}\right)}} \\
& \quad \times\left\|b_{2}\right\|_{*} D_{2}(k, j) v\left(B_{j}\right)^{\alpha_{2} / n}\left\|g \chi_{j}\right\|_{L^{q_{2}\left(\omega_{2}\right)}} .
\end{aligned}
$$

By symmetry, we also get

$$
\begin{aligned}
& v\left(B_{k}\right)^{\alpha / n}\left\|\left(L_{3}\right) \chi_{k}\right\|_{L^{q}\left(v_{\vec{\omega}}\right)} \\
& \leq C\left\|b_{1}\right\|_{*} D_{1}(k, i) v\left(B_{i}\right)^{\alpha_{1} / n}\left\|f \chi_{i}\right\|_{L^{q_{1}\left(\omega_{1}\right)}} \\
& \quad \times\left\|b_{2}\right\|_{*} D_{2}(k, j) v\left(B_{j}\right)^{\alpha_{2} / n}\left\|g \chi_{j}\right\|_{L^{q_{2}\left(\omega_{2}\right)}} .
\end{aligned}
$$

Finally, it remains to estimate $J_{4}$. Applying (29) and Hölder's inequality,

$$
\begin{aligned}
& \left|L_{4}(x)\right|=\left|T\left(\left(b_{1}-\lambda_{1 i}\right) f \chi_{i},\left(b_{2}-\lambda_{2 j}\right) g \chi_{j}\right)\right| \\
& \quad \leq C 2^{-2 \max \{i, j, k\} n}\left\|\left(b_{1}-\lambda_{1 i}\right) f \chi_{i}\right\|_{L^{1}} \\
& \left.\cdot \|\left(b_{2}-\lambda_{2 j}\right) g \chi_{j}\right)\left\|_{L^{1}} \leq C 2^{-2 \max \{i, j, k\} n}\right\| f \chi_{i} \|_{L^{q_{1}}\left(\omega_{1}\right)} \\
& \cdot\left(\int_{B_{i}}\left|b_{1}(x)-\lambda_{1 i}\right|^{q_{1}^{\prime}} \omega_{1}(x)^{1-q_{1}^{\prime}} d x\right)^{1 / q_{1}^{\prime}} \\
& \quad \times\left\|g \chi_{j}\right\|_{L^{q_{2}}\left(\omega_{2}\right)} \\
& \quad\left(\int_{B_{j}}\left|b_{2}(x)-\lambda_{2 j}\right|^{q_{2}^{\prime}} \omega_{2}(x)^{1-q_{2}^{\prime}} d x\right)^{1 / q_{2}^{\prime}} .
\end{aligned}
$$

By (46) and (16),

$$
\begin{aligned}
& \mid L_{4}(x) \leq C\left\|b_{1}\right\|_{*} 2^{-\max \{i, k\} n}\left\|f \chi_{i}\right\|_{L^{q_{1}\left(\omega_{1}\right)}} \\
& \cdot\left(\int_{B_{i}} \omega_{1}(x)^{1-q_{1}^{\prime}} d x\right)^{1 / q_{1}^{\prime}} \times\left\|b_{2}\right\|_{*} \\
& \cdot 2^{-\max \{j, k\} n}\left\|g \chi_{j}\right\|_{L^{q_{2}}\left(\omega_{2}\right)}\left(\int_{B_{j}} \omega_{2}(x)^{1-q_{2}^{\prime}} d x\right)^{1 / q_{2}^{\prime}} \\
& \quad \leq C\left\|b_{1}\right\|_{*} 2^{-\max \{i, k\} n+i n}\left\|f \chi_{i}\right\|_{L^{q_{1}\left(\omega_{1}\right)}} \omega_{1}\left(B_{i}\right)^{-1 / q_{1}} \\
& \quad \times\left\|b_{2}\right\|_{*} 2^{-\max \{j, k\} n+j n}\left\|g \chi_{j}\right\|_{L^{q_{2}\left(\omega_{2}\right)}} \omega_{2}\left(B_{j}\right)^{-1 / q_{2}} .
\end{aligned}
$$


Then

$$
\begin{aligned}
& \nu\left(B_{k}\right)^{\alpha / n}\left\|\left(L_{4}\right) \chi_{k}\right\|_{L^{q}\left(v_{\vec{\omega}}\right)} \leq C\left\|b_{1}\right\|_{*} \\
& \cdot 2^{-\max \{i, k\} n+i n} \nu\left(B_{i}\right)^{\alpha_{1} / n}\left\|f \chi_{i}\right\|_{L^{q_{1}\left(\omega_{1}\right)}}\left(\frac{\nu\left(B_{k}\right)}{\nu\left(B_{i}\right)}\right)^{\alpha_{1} / n} \\
& \cdot\left(\frac{\omega_{1}\left(B_{k}\right)}{\omega_{1}\left(B_{i}\right)}\right)^{1 / q_{1}} \times\left\|b_{2}\right\|_{*} 2^{-\max \{j, k\} n+j n} \nu\left(B_{j}\right)^{\alpha_{2} / n} \\
& \cdot\left\|g \chi_{j}\right\|_{L^{q_{2}\left(\omega_{2}\right)}}\left(\frac{\nu\left(B_{k}\right)}{\nu\left(B_{j}\right)}\right)^{\alpha_{2} / n}\left(\frac{\omega_{2}\left(B_{k}\right)}{\omega_{2}\left(B_{j}\right)}\right)^{1 / q_{2}} \\
& \leq C\left\|b_{1}\right\|_{*}|k-i| 2^{-\max \{i, k\} n+i n} \nu\left(B_{i}\right)^{\alpha_{1} / n}\left\|f \chi_{i}\right\|_{L^{q_{1}\left(\omega_{1}\right)}} \\
& \cdot\left(\frac{\nu\left(B_{k}\right)}{\nu\left(B_{i}\right)}\right)^{\alpha_{1} / n}\left(\frac{\omega_{1}\left(B_{k}\right)}{\omega_{1}\left(B_{i}\right)}\right)^{1 / q_{1}} \times\left\|b_{2}\right\|_{*}|k-j| \\
& \cdot 2^{-\max \{j, k\} n+j n} \nu\left(B_{j}\right)^{\alpha_{2} / n}\left\|g \chi_{j}\right\|_{L^{q_{2}}\left(\omega_{2}\right)}\left(\frac{\nu\left(B_{k}\right)}{\nu\left(B_{j}\right)}\right)^{\alpha_{2} / n} \\
& \cdot\left(\frac{\omega_{2}\left(B_{k}\right)}{\omega_{2}\left(B_{j}\right)}\right)^{1 / q_{2}} \cdot
\end{aligned}
$$

Thus

$$
\begin{aligned}
& v\left(B_{k}\right)^{\alpha / n}\left\|\left(L_{4}\right) \chi_{k}\right\|_{L^{q}\left(v_{\vec{\omega}}\right)} \\
& \leq C\left\|b_{1}\right\|_{*} D_{1}(k, i) v\left(B_{i}\right)^{\alpha_{1} / n}\left\|f \chi_{i}\right\|_{L^{q_{1}}\left(\omega_{1}\right)} \\
& \times\left\|b_{2}\right\|_{*} D_{2}(k, j) v\left(B_{j}\right)^{\alpha_{2} / n}\left\|g \chi_{j}\right\|_{L^{q_{2}}\left(\omega_{2}\right)} .
\end{aligned}
$$

Now, we give the proof of Theorem 1. have

If $q>1$, by Minkowskis inequality and Theorem 5, we

$$
\begin{aligned}
& v\left(B_{k}\right)^{\alpha / n}\left\|T_{\Pi b}(f, g) \chi_{k}\right\|_{L^{q}\left(\gamma_{\vec{\omega}}\right)} \\
& \leq C \nu\left(B_{k}\right)^{\alpha / n}\left\|\sum_{i=-\infty}^{\infty} \sum_{j=-\infty}^{\infty} T_{\Pi b}\left(f \chi_{i}, g \chi_{j}\right) \chi_{k}\right\|_{L^{q}\left(\nu_{\vec{\omega}}\right)} \\
& \leq C \sum_{i=-\infty}^{\infty} \sum_{j=-\infty}^{\infty} v\left(B_{k}\right)^{\alpha / n}\left\|T_{\Pi b}\left(f \chi_{i}, g \chi_{j}\right) \chi_{k}\right\|_{L^{q}\left(\nu_{\vec{\omega}}\right)} \\
& \leq C\left\|b_{1}\right\|_{*}\left\|b_{2}\right\|_{*} \sum_{i=-\infty}^{\infty} D_{1}(k, i) v\left(B_{i}\right)^{\alpha_{1} / n}\left\|f \chi_{i}\right\|_{L^{q_{1}\left(\omega_{1}\right)}} \\
& \quad \times \sum_{j=-\infty}^{\infty} D_{2}(k, j) v\left(B_{j}\right)^{\alpha_{2} / n}\left\|g \chi_{j}\right\|_{L^{q_{2}\left(\omega_{2}\right)}} \\
& \leq C\left\|b_{1}\right\|_{*}\left\|b_{2}\right\|_{*} \sum_{i=-\infty}^{\infty} D_{1}(k, i)^{1-\epsilon} v\left(B_{i}\right)^{\alpha_{1} / n}\left\|f \chi_{i}\right\|_{L^{q_{1}}\left(\omega_{1}\right)} \\
& \quad \times \sum_{j=-\infty}^{\infty} D_{2}(k, j)^{1-\epsilon} v\left(B_{j}\right)^{\alpha_{2} / n}\left\|g \chi_{j}\right\|_{L^{q_{2}}\left(\omega_{2}\right)}
\end{aligned}
$$

for any $0<\epsilon<1$, since $D_{1}(k, i)+D_{2}(k, j) \rightarrow 0$ whenever $i, j \rightarrow \pm \infty$.

If $0<q \leq 1$, note the fact that

$$
\begin{aligned}
& \sum_{i=-\infty}^{\infty} D_{1}(k, i)^{q \epsilon /(1-q)}<\infty, \\
& \sum_{j=-\infty}^{\infty} D_{1}(k, j)^{q \epsilon /(1-q)}<\infty,
\end{aligned}
$$

and then by Theorem 5 , the inequality $\left(\sum\left|a_{i}\right|\right)^{q} \leq \sum\left|a_{i}\right|^{q}$, and Hölder's inequality, we have

$$
\begin{aligned}
v & \left(B_{k}\right)^{\alpha / n}\left\|T_{\Pi b}(f, g) \chi_{k}\right\|_{L^{q}\left(\gamma_{\tilde{\omega}}\right)} \\
& \leq C\left\{\sum_{i=-\infty}^{\infty} \sum_{j=-\infty}^{\infty}\left[v\left(B_{k}\right)^{\alpha / n}\left\|T_{\Pi b}\left(f \chi_{i}, g \chi_{j}\right) \chi_{k}\right\|_{L^{q}\left(\nu_{\tilde{\omega}}\right)}\right]^{q}\right\}^{1 / q} \\
& \leq C\left\|b_{1}\right\|_{*}\left\|b_{2}\right\|_{*}\left\{\sum_{i=-\infty}^{\infty} D_{1}(k, i)^{q} \nu\left(B_{i}\right)^{\alpha_{1} q / n}\left\|f \chi_{i}\right\|_{L^{q_{1}\left(\omega_{1}\right)}}^{q}\right. \\
& \left.\times \sum_{j=-\infty}^{\infty} D_{2}(k, j)^{q} \nu\left(B_{j}\right)^{\alpha_{2} q / n}\left\|g \chi_{j}\right\|_{L^{q_{2}\left(\omega_{2}\right)}}^{q}\right\}^{1 / q} \cdot \leq C\left\|b_{1}\right\|_{*} \\
& \cdot\left\|b_{2}\right\|_{*} \sum_{i=-\infty}^{\infty} D_{1}(k, i)^{1-\epsilon} \nu\left(B_{i}\right)^{\alpha_{1} / n}\left\|f \chi_{i}\right\|_{L^{q_{1}}\left(\omega_{1}\right)} \\
& \cdot\left\{\sum_{j=-\infty}^{\infty} D_{1}(k, j)^{q \epsilon /(1-q)}\right\}^{(1-q) / q} \\
& \times \sum_{j=-\infty}^{\infty} D_{2}(k, j)^{1-\epsilon} \nu\left(B_{j}\right)^{\alpha_{2} / n}\left\|g \chi_{j}\right\|_{L^{q_{2}}\left(\omega_{2}\right)} \\
& \cdot\left\{\sum_{j=-\infty}^{\infty} D_{2}(k, i)^{q \epsilon /(1-q)}\right\}^{(1-q) / q} \leq C\left\|b_{1}\right\|_{*}\left\|b_{2}\right\|_{*} \\
& \cdot \sum_{i=-\infty}^{\infty} D_{1}(k, i)^{1-\epsilon} v\left(B_{i}\right)^{\alpha_{1} / n}\left\|f \chi_{i}\right\|_{L^{q_{1}}\left(\omega_{1}\right)} \\
& \times \sum_{j=-\infty}^{\infty} D_{2}(k, j)^{1-\epsilon} \nu\left(B_{j}\right)^{\alpha_{2} / n}\left\|g \chi_{j}\right\|_{L^{q_{2}}\left(\omega_{2}\right)} .
\end{aligned}
$$

Since $0<p<\infty$, by (56), (58) and Hölder's inequality,

$$
\begin{gathered}
\left\|T_{\Pi b}(f, g)\right\|_{\dot{K}_{q}^{\alpha, p}\left(v, v_{\tilde{\omega}}\right)\left(\mathbb{R}^{n}\right)} \leq\left\{\sum_{k=-\infty}^{\infty} v\left(B_{k}\right)^{\alpha p / n}\right. \\
\left.\cdot\left\|T_{\Pi b}(f, g) \chi_{k}\right\|_{L^{q}\left(\gamma_{\tilde{\omega}}\right)}^{p}\right\}^{1 / p} \leq C\left\|b_{1}\right\|_{*}\left\|b_{2}\right\|_{*} \\
\cdot\left\{\sum _ { k = - \infty } ^ { \infty } \left(\sum_{i=-\infty}^{\infty} D_{1}(k, i)^{1-\epsilon} \nu\left(B_{i}\right)^{\alpha_{1} / n}\left\|f \chi_{i}\right\|_{L^{q_{1}}\left(\omega_{1}\right)}\right.\right.
\end{gathered}
$$


$\left.\left.\times \sum_{j=-\infty}^{\infty} D_{2}(k, j)^{1-\epsilon} v\left(B_{j}\right)^{\alpha_{2} / n}\left\|g \chi_{j}\right\|_{L^{q_{2}}\left(\omega_{2}\right)}\right)^{p}\right\}^{1 / p} \leq C\left\|b_{1}\right\|_{*}$

$\cdot\left\|b_{2}\right\|_{*}$

$\cdot\left\{\sum_{k=-\infty}^{\infty}\left(\sum_{i=-\infty}^{\infty} D_{1}(k, i)^{1-\epsilon} v\left(B_{i}\right)^{\alpha_{1} / n}\left\|f \chi_{i}\right\|_{L^{q_{1}}\left(\omega_{1}\right)}\right)^{p_{1}}\right\}^{1 / p_{1}}$
$\times\left\{\sum_{k=-\infty}^{\infty}\left(\sum_{j=-\infty}^{\infty} D_{2}(k, j)^{1-\epsilon} v\left(B_{j}\right)^{\alpha_{2} / n}\left\|g \chi_{j}\right\|_{L^{q_{2}\left(\omega_{2}\right)}}\right)^{p_{2}}\right\}^{1 / p_{2}}$

$=C\left\|b_{1}\right\|_{*}\left\|b_{2}\right\|_{*} N_{1} \times N_{2}$.

It is enough to show that $N_{1} \leq C\|f\|_{\dot{K}_{q_{1}}^{\alpha_{1}, p_{1}}\left(v, \omega_{1}\right)\left(\mathbb{R}^{n}\right)}$ and $N_{2} \leq$ $C\|g\|_{\dot{K}_{q_{2}}^{\alpha_{2}, p_{2}}\left(v, \omega_{2}\right)\left(\mathbb{R}^{n}\right)}$. By the symmetry, we only give the estimate for $N_{1}$. Consider

$$
\begin{aligned}
& N_{1}^{p_{1}} \\
& \leq \sum_{k=-\infty}^{\infty}\left(\sum_{i=-\infty}^{k-2} D_{1}(k, i)^{1-\epsilon} v\left(B_{i}\right)^{\alpha_{1} / n}\left\|f \chi_{i}\right\|_{L^{q_{1}\left(\omega_{1}\right)}}\right)^{p_{1}} \\
& \quad+\sum_{k=-\infty}^{\infty}\left(\sum_{i=k-1}^{k+1} D_{1}(k, i)^{1-\epsilon} v\left(B_{i}\right)^{\alpha_{1} / n}\left\|f \chi_{i}\right\|_{L^{q_{1}}\left(\omega_{1}\right)}\right)^{p_{1}} \\
& \quad+\sum_{k=-\infty}^{\infty}\left(\sum_{i=k+2}^{\infty} D_{1}(k, i)^{1-\epsilon} v\left(B_{i}\right)^{\alpha_{1} / n}\left\|f \chi_{i}\right\|_{L^{q_{1}}\left(\omega_{1}\right)}\right)^{p_{1}} \\
& =N_{11}+N_{12}+N_{13} .
\end{aligned}
$$

If $0<p_{1} \leq 1$, then, by the inequality $\left(\sum\left|a_{i}\right|\right)^{p_{1}} \leq \sum\left|a_{i}\right|^{p_{1}}$ and the fact that

$$
\begin{aligned}
& \sum_{k=i+2}^{\infty} D_{1}(k, i)^{(1-\epsilon) p_{1}} \\
& \quad=\sum_{k=i+2}^{\infty}(k-i)^{(1-\epsilon) p_{1}} 2^{(1-\epsilon) p_{1}(k-i)\left(\alpha_{1} r+n r_{1} / q_{1}-n\right)}<\infty,
\end{aligned}
$$

we get

$$
\begin{aligned}
N_{11} & \leq \sum_{i=-\infty}^{\infty} v\left(B_{i}\right)^{\alpha_{1} p_{1} / n}\left\|f \chi_{i}\right\|_{L^{q_{1}\left(\omega_{1}\right)}}^{p_{1}} \sum_{k=i+2}^{\infty} D_{1}(k, i)^{(1-\epsilon) p_{1}} \\
& \leq C \sum_{i=-\infty}^{\infty} v\left(B_{i}\right)^{\alpha_{1} p_{1} / n}\left\|f \chi_{i}\right\|_{L^{q_{1}}\left(\omega_{1}\right)}^{p_{1}} \\
& \leq C\|f\|_{\dot{K}_{q_{1}}^{\alpha_{1}, p_{1}}\left(v, \omega_{1}\right)\left(\mathbb{R}^{n}\right)}^{p_{1}}
\end{aligned}
$$

Note that

$$
\begin{aligned}
& \sum_{i=-\infty}^{k-2} D_{1}(k, i)^{1-\epsilon} \\
& \quad=\sum_{k=i+2}^{\infty} D_{1}(k, i)^{1-\epsilon} \\
& \quad \leq C \sum_{k=i+2}^{\infty}(k-i)^{(1-\epsilon)} 2^{(1-\epsilon)(k-i)\left(\alpha_{1} r+n r_{1} / q_{1}-n\right)}<\infty .
\end{aligned}
$$

Then, for $p_{1}>1$, we have

$$
\begin{aligned}
& N_{11} \leq C \sum_{k=-\infty}^{\infty}\left(\sum_{i=-\infty}^{k-2} D_{1}(k, i)^{1-\epsilon} v\left(B_{i}\right)^{\alpha_{1} p_{1} / n}\right. \\
& \left.\cdot\left\|f \chi_{i}\right\|_{L^{q_{1}}\left(\omega_{1}\right)}^{p_{1}}\right)\left(\sum_{i=-\infty}^{k-2} D_{1}(k, i)^{1-\epsilon}\right)^{p_{1} / p_{1}^{\prime}} \\
& \leq C \sum_{k=-\infty}^{\infty} \sum_{i=-\infty}^{k-2} D_{1}(k, i)^{1-\epsilon} v\left(B_{i}\right)^{\alpha_{1} p_{1} / n}\left\|f \chi_{i}\right\|_{L^{q_{1}}\left(\omega_{1}\right)}^{p_{1}} \\
& \leq C \sum_{i=-\infty}^{\infty} v\left(B_{i}\right)^{\alpha_{1} p_{1} / n}\left\|f \chi_{i}\right\|_{L^{q_{1}}\left(\omega_{1}\right)}^{p_{p_{1}}} \sum_{k=i+2}^{\infty} D_{1}(k, i)^{1-\epsilon} \\
& \leq C\|f\|_{\dot{K}_{q_{1}}^{\alpha_{1}, p_{1}}\left(v, \omega_{1}\right)\left(\mathbb{R}^{n}\right)}^{p_{1}} .
\end{aligned}
$$

Since $D_{1}(k, i)=1$ for $i=k-1, k, k+1$, then

$$
\begin{aligned}
N_{12} & \leq C \sum_{k=-\infty}^{\infty} \sum_{i=k-1}^{k+1} v\left(B_{i}\right)^{\alpha_{1} p_{1} / n}\left\|f \chi_{i}\right\|_{L^{q_{1}}\left(\omega_{1}\right)}^{p_{1}} \\
& \leq C \sum_{i=-\infty}^{\infty} \sum_{k=i-1}^{i+1} v\left(B_{i}\right)^{\alpha_{1} p_{1} / n}\left\|f \chi_{i}\right\|_{L^{q_{1}}\left(\omega_{1}\right)}^{p_{1}} \\
& \leq C \sum_{i=-\infty}^{\infty} v\left(B_{i}\right)^{\alpha_{1} p_{1} / n}\left\|f \chi_{i}\right\|_{L^{q_{1}}\left(\omega_{1}\right)}^{p_{1}} \\
& \leq C\|f\|_{\dot{K}_{q_{1}}^{\alpha_{1}, p_{1}}\left(v, \omega_{1}\right)\left(\mathbb{R}^{n}\right)}^{p_{1}} .
\end{aligned}
$$

Thus, we have obtained that $N_{12} \leq C\|f\|_{\dot{K}_{q_{1}}^{\alpha_{1}, p_{1}}\left(v, \omega_{1}\right)\left(\mathbb{R}^{n}\right)}^{p_{1}}$ holds for any $0<p_{1}<\infty$.

Let us now turn to estimate the last term $N_{13}$. If $0<p_{1} \leq$ 1 , then

$N_{13}$

$$
\begin{aligned}
& \leq C \sum_{k=-\infty}^{\infty} \sum_{i=k+2}^{\infty} D_{1}(k, i)^{(1-\epsilon) p_{1}} v\left(B_{i}\right)^{\alpha_{1} p_{1} / n}\left\|f \chi_{i}\right\|_{L^{q_{1}}\left(\omega_{1}\right)}^{p_{1}} \\
& \leq C \sum_{i=-\infty}^{\infty} \nu\left(B_{i}\right)^{\alpha_{1} p_{1} / n}\left\|f \chi_{i}\right\|_{L^{q_{1}\left(\omega_{1}\right)}}^{p_{1}} \sum_{k=-\infty}^{i-2} D_{1}(k, i)^{(1-\epsilon) p_{1}} .
\end{aligned}
$$


But

$$
\begin{aligned}
& \sum_{k=-\infty}^{i-2} D_{1}(k, i)^{(1-\epsilon) p_{1}} \\
& \quad=\sum_{k=-\infty}^{i-2}(i-k)^{(1-\epsilon) p_{1}} 2^{(1-\epsilon) p_{1}(k-i)\left(\delta \alpha_{1} / n+\delta_{1} / q_{1}\right)}<\infty .
\end{aligned}
$$

Then

$$
\begin{aligned}
N_{13} & \leq C \sum_{i=-\infty}^{\infty} v\left(B_{i}\right)^{\alpha_{1} p_{1} / n}\left\|f \chi_{i}\right\|_{L^{q_{1}}\left(\omega_{1}\right)}^{p_{1}} \\
& \leq C\|f\|_{\dot{K}_{q_{1}}^{\alpha_{1}, p_{1}}\left(v, \omega_{1}\right)\left(\mathbb{R}^{n}\right)}^{p_{1}} .
\end{aligned}
$$

Note that

$$
\begin{aligned}
& \sum_{k=-\infty}^{i-2} D_{1}(k, i)^{1-\epsilon} \\
& \quad=\sum_{i=k+2}^{\infty} D_{1}(k, i)^{1-\epsilon} \\
& \quad \leq C \sum_{i=k+2}^{\infty}(i-k)^{1-\epsilon} 2^{(1-\epsilon)(k-i)\left(\delta \alpha_{1} / n+\delta_{1} / q_{1}\right)}<\infty .
\end{aligned}
$$

Then, for $p>1$, we have

$$
\begin{aligned}
& N_{13} \leq C \sum_{k=-\infty}^{\infty}\left(\sum_{i=k+2}^{\infty} D_{1}(k, i)^{1-\epsilon} v\left(B_{i}\right)^{\alpha_{1} p_{1} / n}\right. \\
& \left.\cdot\left\|f \chi_{i}\right\|_{L^{q_{1}}\left(\omega_{1}\right)}^{p_{1}}\right)\left(\sum_{i=k+2}^{\infty} D_{1}(k, i)^{1-\epsilon}\right)^{p_{1} / p_{1}^{\prime}} \\
& \leq C \sum_{k=-\infty}^{\infty} \sum_{i=k+2}^{\infty} D_{1}(k, i)^{1-\epsilon} v\left(B_{i}\right)^{\alpha_{1} p_{1} / n}\left\|f \chi_{i}\right\|_{L^{q_{1}}\left(\omega_{1}\right)}^{p_{1}} \\
& \leq C \sum_{i=-\infty}^{\infty} v\left(B_{i}\right)^{\alpha_{1} p_{1} / n}\left\|f \chi_{i}\right\|_{L^{q_{1}}\left(\omega_{1}\right)}^{p_{1}} \sum_{k=-\infty}^{i-2} D_{1}(k, i)^{1-\epsilon} \\
& \leq C\|f\|_{\dot{K}_{q_{1}}^{\alpha_{1}, p_{1}}\left(v, \omega_{1}\right)\left(\mathbb{R}^{n}\right)}^{p_{1}} \cdot
\end{aligned}
$$

This completes the proof of Theorem 1 .

Remark 6. Using Theorem 5 and the methods in [18], we can easily get the boundedness of the iterated commutator $T_{\Pi b}$ on weighted Herz-Morrey spaces.

\section{Conflict of Interests}

The authors declare that there is no conflict of interests regarding the publication of this paper.

\section{Acknowledgments}

The authors are very grateful to the anonymous referees and the editor for their insightful comments and suggestions.

\section{References}

[1] R. R. Coifman and Y. Meyer, "On commutators of singular integrals and bilinear singular integrals," Transactions of the American Mathematical Society, vol. 212, pp. 315-331, 1975.

[2] L. Grafakos and R. H. Torres, "Multilinear Calderón-Zygmund theory," Advances in Mathematics, vol. 165, no. 1, pp. 124-164, 2002.

[3] L. Grafakos and R. H. Torres, "Maximal operator and weighted norm inequalities for multilinear singular integrals," Indiana University Mathematics Journal, vol. 51, no. 5, pp. 1261-1276, 2002.

[4] L. Grafakos and R. Torres, "On multilinear singular integrals of Calderón-Zygmund type," Publicacions Matemàtiques, vol. 46, Extra, pp. 57-91, 2002.

[5] A. K. Lerner, S. Ombrosi, C. Pérez, R. H. Torres, and R. TrujilloGonzález, "New maximal functions and multiple weights for the multilinear Calderón-Zygmund theory," Advances in Mathematics, vol. 220, no. 4, pp. 1222-1264, 2009.

[6] C. Pérez, G. Pradolini, R. H. Torres, and R. Trujillo-González, "End-point estimates for iterated commutators of multilinear singular integrals," Bulletin of the London Mathematical Society, vol. 46, no. 1, pp. 26-42, 2014.

[7] G. Hu and Y. Zhu, "Weighted norm inequalities for the commutators of multilinear singular integral operators," Acta Mathematica Scientia. Series B. English Edition, vol. 31, no. 3, pp. 749-764, 2011.

[8] G. Hu and Y. Meng, "Estimates for multilinear singular integral operators with nonsmooth kernels," Frontiers of Mathematics in China, vol. 7, no. 1, pp. 51-67, 2012.

[9] C. Pérez and R. H. Torres, "Sharp maximal function estimates for multilinear singular integrals," Contemporary Mathematics, vol. 320, pp. 323-331, 2003.

[10] L. Tang, "Weighted estimates for vector-valued commutators of multilinear operators," Proceedings of the Royal Society of Edinburgh Section A: Mathematics, vol. 138, no. 4, pp. 897-922, 2008.

[11] S. Z. Lu and D. C. Yang, "The decomposition of weighted Herz space on $\mathbb{R}^{n}$ and its applications," Science in China (Scientia Sinica). Series A. Mathematics, Physics, Astronomy, vol. 38, no. 2, pp. 147-158, 1995.

[12] S. Lu, K. Yabuta, and D. Yang, "Boundedness of some sublinear operators in weighted Herz-type spaces," Kodai Mathematical Journal, vol. 23, no. 3, pp. 391-410, 2000.

[13] H. Wang, "The boundedness of intrinsic square functions on the weighted Herz spaces," Journal of Function Spaces, vol. 2014, Article ID 274521, 14 pages, 2014.

[14] H. Wang, "Some estimates of intrinsic square functions on the weighted Herz-type HARdy spaces," Journal of Inequalities and Applications, vol. 62, 22 pages, 2015.

[15] J. Kuang, "Generalized Hausdorff operators on weighted HERz spaces," Matematichki Vesnik, vol. 66, no. 1, pp. 19-32, 2014.

[16] Y. Hu, Y. He, and Y. Wang, "The commutators of fractional integrals on generalized Herz spaces," Journal of Function Spaces, vol. 2014, Article ID 428493, 6 pages, 2014.

[17] G. Tao, "Boundedness of multilinear operators on weighted Herz type spaces," Analysis in Theory and Applications, vol. 21, no. 1, pp. 73-89, 2005.

[18] X. Tao and H. Zhang, "On the boundedness of multilinear operators on weighted Herz-Morrey spaces," Taiwanese Journal of Mathematics, vol. 15, no. 4, pp. 1527-1543, 2011. 
[19] A. Hussain and G. Gao, "Multilinear singular integrals and commutators on Herz space with variable exponent," ISRN Mathematical Analysis, vol. 2014, Article ID 626327, 10 pages, 2014.

[20] B. Muckenhoupt, "Weighted norm inequalities for the Hardy maximal function," Transactions of the American Mathematical Society, vol. 165, pp. 207-226, 1972.

[21] J. Garca-Cuerva and J. R. de Francia, Weighted Norm Inequalities and Related Topics, North-Holland, Amsterdam, The Netherlands, 1985.

[22] Y. Hu and Y. Wang, "Multilinear fractional integral operators on generalized weighted Morrey spaces," Journal of Inequalities and Applications, vol. 2014, article 323, 18 pages, 2014. 




Advances in

Operations Research

mansans

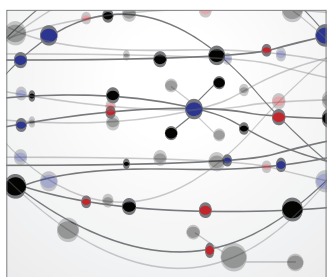

The Scientific World Journal
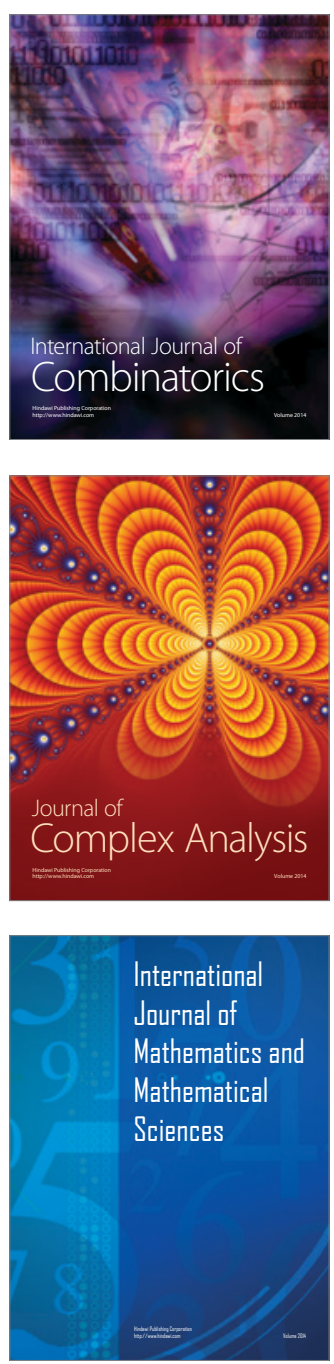
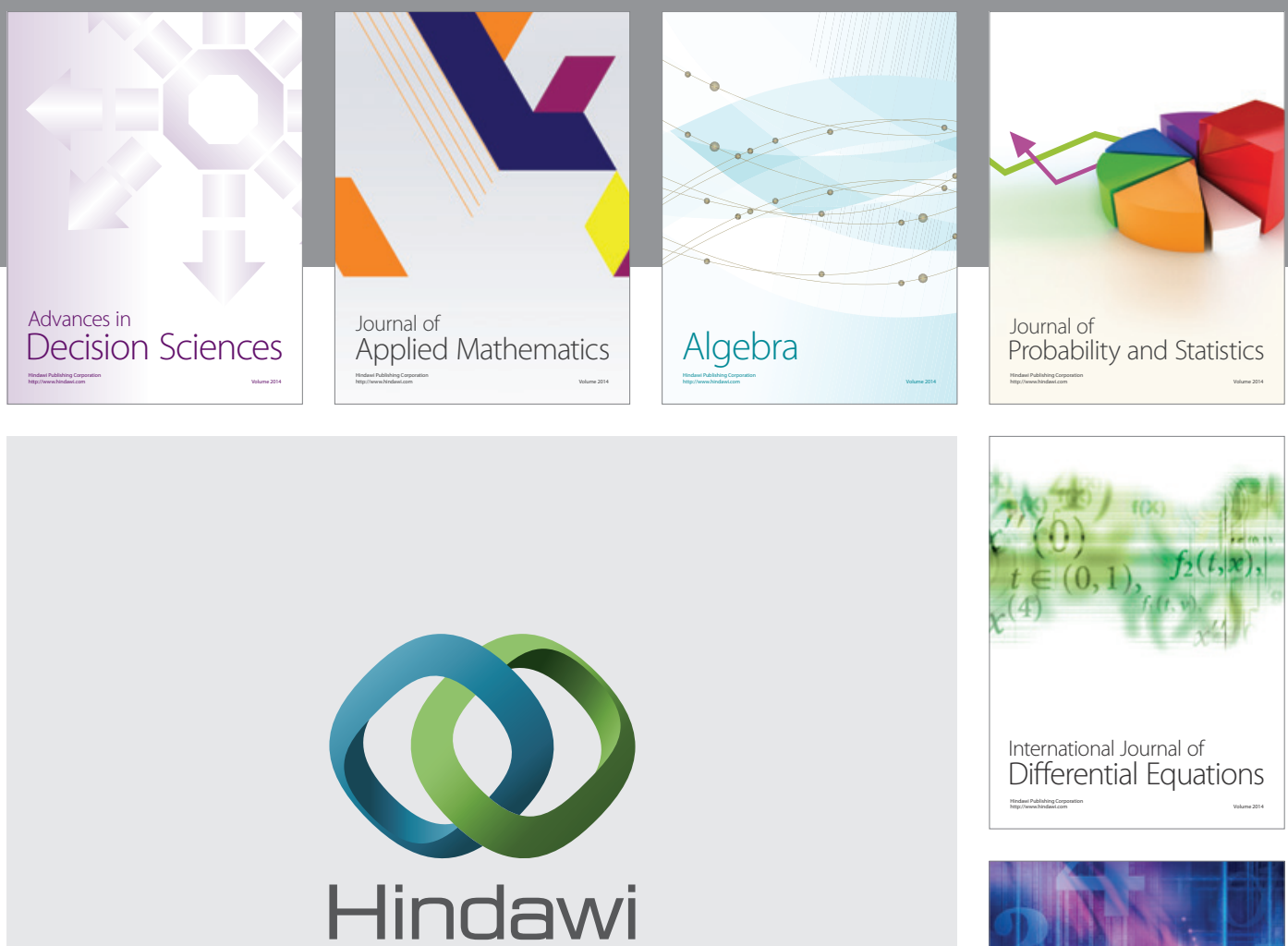

Submit your manuscripts at http://www.hindawi.com
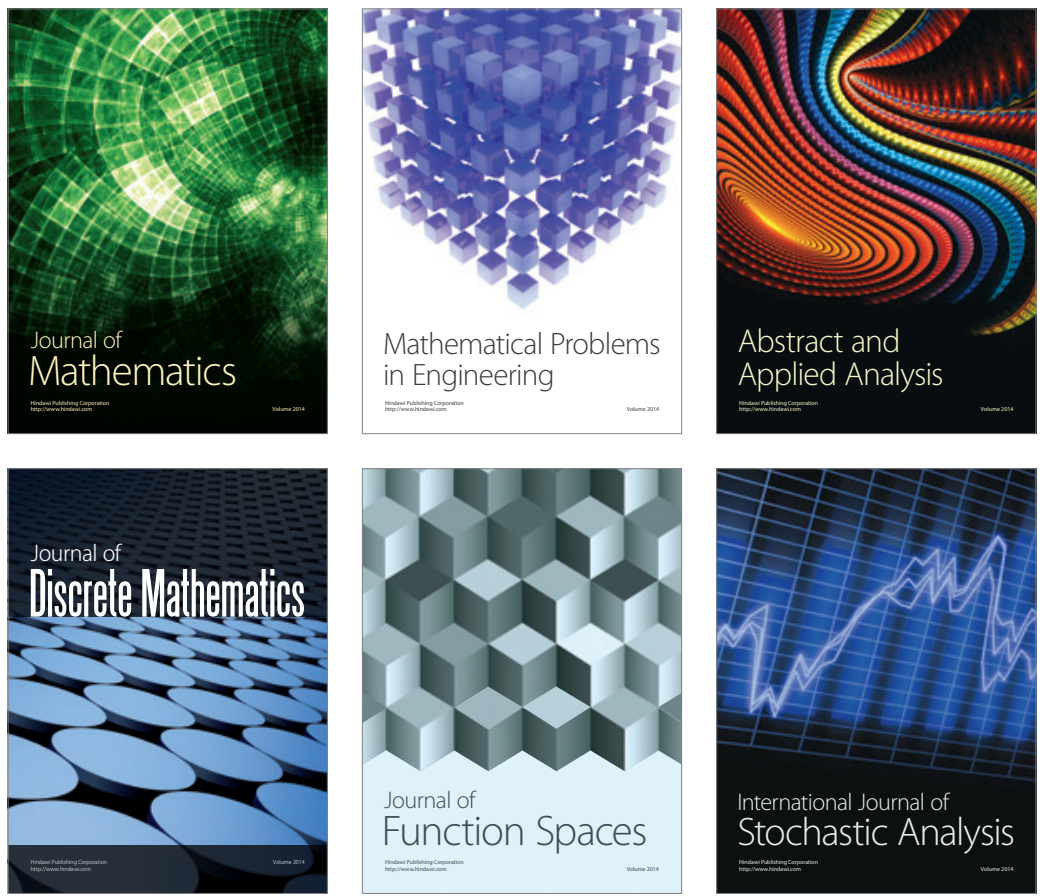

Journal of

Function Spaces



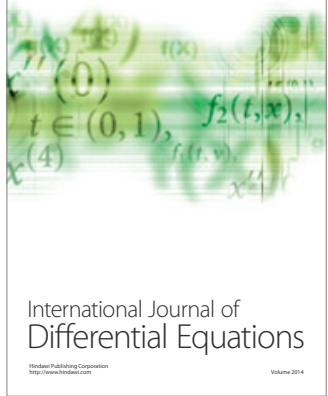
